\title{
AN OBJECT STATE ESTIMATION FOR THE PEG TRANSFER TASK IN COMPUTER-GUIDED SURGICAL TRAINING
}

\author{
Kai Meisner \\ Department of Computer Science \\ Universität der Bundeswehr \\ Werner-Heisenberg-Weg 39, \\ 85579, Neubiberg, Germany \\ kai.meisner@googlemail.com
}

\author{
Minsik Hong \\ Jerzy W. Rozenblit \\ Department of Electrical and Computer \\ Engineering \\ University of Arizona \\ 1230 E Speedway Boulevard, Tucson, AZ, USA \\ mshong@email.arizona.edu; jr@ece.arizona.edu
}

\begin{abstract}
Computer-based simulators have been developed to enhance training experiences in laparoscopic surgical skills training. Most simulators can evaluate a trainee's performance objectively. However, only few simulators can provide active guidance features such as audio and visual guidance. In this paper, an object state estimation and tracking method is presented to support visual and force guidance for computer-assisted surgical trainer (CAST) using image processing schemes in real-time fashion given a specific object transfer task. The experimental results show that the proposed tracking method reaches 100 frame per seconds and estimates an object state effectively for the standard laparoscopy peg transfer task.
\end{abstract}

Keywords: simulation-based surgical training, object recognition, object state detection.

\section{INTRODUCTION}

Minimally invasive surgery (MIS), also known as laparoscopic surgery, has been widely performed since the late 1980s (Lau, Leow, and Li 1997). It reduces recovery time and minimizes blood-loss and postoperative pain. However, this surgical procedure is more challenging than open surgery due to the need for special surgical instruments, which causes restricted vision and lack of both tactile feedback and depth perception. As a standard training program, the Fundamentals of Laparoscopic Surgery (FLS) ("Fundamentals of Laparoscopic Surgery" 2019) was established to teach the fundamental knowledge and motor skills, and to assess trainees in their acquisition of skills by providing an web-based education module and hands-on exams.

Simulation-based training systems have been developed to enhance training procedures in non-patient based setting. Simple box trainers which are generally portable and physical-model based setups were firstly introduced (Soper and Fried 2008). Computer-based simulators (Stylopoulos et al. 2004; Escamirosa et al. 2015; Rosen et al. 2002; Duffy et al. 2005; Salkini et al. 2010; Lahanas et al. 2015) have also been proposed to provide a better training experience and to assess trainees' performance objectively. To present training tasks, the computer-based simulators can use physical reality (PR), virtual reality (VR), or augmented reality (AR) setups. Using these training systems, trainees can practice basic motor skills such as instrument navigation, object transferring, cutting, and suturing.

Most computer-based simulators can evaluate a trainee's performance using objective assessment metrics (Oropesa et al. 2011; Escamirosa et al. 2015) given a specific training exercise. However, only few 
simulators provide active guidance (e.g., instant feedback to teach proper instrument movements) methods to teach trainees (Hernansanz et al. 2012; Howard and Szewczyk 2014; Tagawa et al. 2013). In (Rozenblit et al. 2014), an approach for the Computer-Assisted Surgical Trainer (CAST) was proposed to provide haptic force (Hong and Rozenblit 2016a) and visual guidance (Wagner and Rozenblit 2017), and objective assessments (Hong, Rozenblit, and Hamilton 2017) given a training setup realized by PR or AR. For instrument navigation tasks, a trainee is asked to move an instrument from one location to another without collisions between the instrument and training environments. To support visual and force guidance, a path generator was proposed to suggest collision free and shortest recommended instrument movement trajectories (Rao, Valenzula, and Rozenblit 2017; Napalkova et al. 2014). For object transferring tasks, the object movements should be recognized and tracked in addition to tracking the instrument movements, to support the guidance system. In this paper, we present an object state estimation and tracking method to provide active guidance features in real-time fashion given a specific object transfer task.

\section{PROBLEM DEFINITION}

FLS has five hands-on exams - peg transfer, precision cutting, ligating loop, and suturing with both intracorporeal and extracorporeal knots. Among these five exams, the peg transfer is selected as a target application for the CAST system. The previous modeling approach of the peg transfer task was reviewed and our problem statement is presented to support the guidance system.

\subsection{Modeling of Peg Transfer Task}

Trainees use two grasper-type surgical instruments to transfer six triangle shaped rubber rings given a peg board which has twelve pegs. A trainee is asked to perform this task within 300 seconds without dropping any objects. The entire task can be decomposed using unit actions. In (Hong and Rozenblit 2016b), five actions - move, grasp, carry, transfer, and place - were modeled to describe this task. Each action can be described as follows:

- $\operatorname{move}(I, P)$ : move an instrument $(I)$ to a location $(P)$

- $\operatorname{grasp}(\mathrm{T}, P, I)$ : grasp a triangle $(\mathrm{T})$ at a location $(P)$ using an instrument $(I)$

- $\operatorname{carry}(\mathrm{T}, P, I)$ : carry a triangle $(\mathrm{T})$ to a location $(P)$ using an instrument $(I)$

- $\operatorname{transfer}(\mathrm{T}, P, I)$ : transfer a triangle $(\mathrm{T})$ at a location $(P)$ from an instrument $(I)$ to another

- $\operatorname{place}(\mathrm{T}, P, I)$ : place a triangle $(\mathrm{T})$ at a location $(P)$ using an instrument $(I)$

where $I \in\{$ left,right $\}$, T is one of 6 triangles, and $P \in \mathbb{R}^{3}$.

By introducing states of objects (e.g., grasper's state), precondition and effects of each action can be defined. Consider the carry action as an example.

$$
\operatorname{carry}(\mathrm{T}, P, I):\left\{\begin{array}{l}
\text { precondition: holding }(\mathrm{T}, I) \\
\text { effects: } \operatorname{obj} \operatorname{AtLoc}(\mathrm{T}, P) \wedge \operatorname{obj} \operatorname{AtLoc}(I, P),
\end{array}\right.
$$

where holding represents that the grasper of the instrument $I$ holds an object T and objAtLoc represents that an object (T or $I$ ) is located at a location $(P)$. Before applying the carry action, the precondition should be satisfied and the state of objects will be updated after the corresponding action is performed by a trainee.

\subsection{Problem Statement}

CAST aims to improve the training experience of a trainee by providing visual, audio, and force guidance. To meet this goal, the object state estimation method is required to support guidance schemes given a specific training scenario. To estimate a state of the object, the object's positions as well as relationships among other objects have to be defined. In (Peng et al. 2019), a single shot state detection (SSSD) method was proposed for the CAST system using a convolutional neural network (CNN). A graphics processing unit (GPU) was essential to use SSSD for a real-time application (i.e., 50 frames per second (FPS) under 
GPU configuration; 12.5 FPS while running on a central processing unit (CPU)). To provide a cost effective solution and support higher frame rates at the same time, we propose an image processing-based approach to reach a benchmark of 100 FPS while running on a CPU given a peg transfer task (PTT) setup.

While a trainee performs the PTT, he or she picks one specific triangle using a grasper, transfers it to another grasper, and places the object on a specific peg (i.e., only one triangle is carried at a time). To provide guidance information, the CAST system should know the initial positions of all triangles as well as their colors. Also, the transition conditions are essential to determine the completion of the action given a series of actions. In (Hong and Rozenblit 2016b), a transition condition for each action was not defined. To define the transition condition, we introduce the state of the triangle as follows:

$$
\text { Triangle state: }\left\{\begin{array}{l}
\operatorname{picked}(\mathrm{T}): A \text { triangle } \mathrm{T} \text { is picked } \\
\neg \text { picked }(\mathrm{T}): A \text { triangle } \mathrm{T} \text { is placed }
\end{array}\right. \text {. }
$$

By combining the state of the triangle with the object positions, a transition condition can be determined and used to express an action. For instance, if a triangle is picked up (picked(T)) and the triangle is close to the grasper while performing a grasp action, we can conclude that the grasp action is completed. When this completion of the action is recognized, the CAST system can update the proper guidance information (e.g., update a recommended trajectory). We apply image processing techniques to predict the position of the triangle in the 2D image coordinate and the triangle state. Also, the triangle should be tracked to estimate an object state which was already defined previously. For instance, the CAST system keeps tracking both the triangle and instrument to estimate the holding $(\mathrm{T}, I)$ state. Our proposed approach is based on the assumption that the object's color is known as is its initial position.

In summary, the objectives of this paper are as follows:

- Design the estimation method and run it on a CPU to reach 100 FPS,

- Track a triangle in the 2D image coordinate,

- Estimate a triangle state (picked or $\neg$ picked).

\subsection{Physical Configuration of Peg Transfer Task}

Two grasper-type instruments, one web camera to imitate a laparoscope, and one peg board with six triangles were used as shown in Figure 1-(a). We can use maximum four different colors - red, green, yellow, and orange. Therefore, some colors have to be used more than once. The camera captures the entire training scene and the pegboard is located at the center of the captured image. Also, the camera is placed above the peg board. Thus, if a triangle is placed on a peg, its distance from the camera becomes maximum as shown in Figure 1-(b). By picking up a triangle, the distance decreases (Figure 1-(c)). If a triangle is picked up, it is closer to the camera and appears larger in the $2 \mathrm{D}$-image.

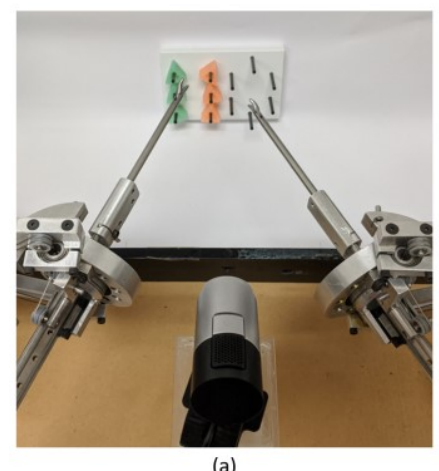

(a)

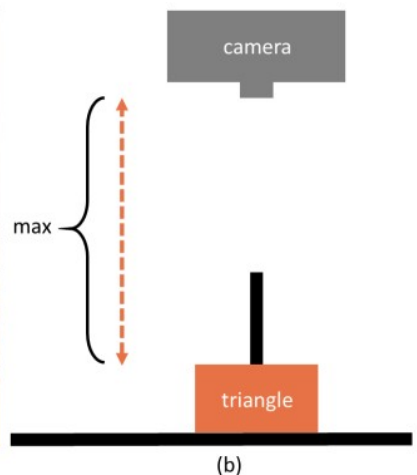

(b)

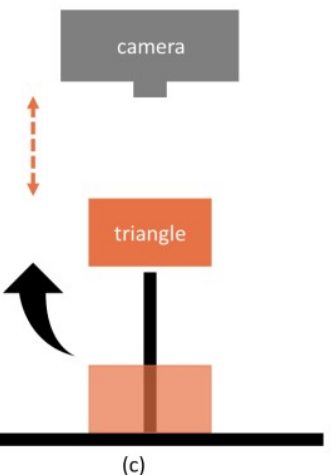

(c)

Figure 1: (a) physical configuration, (b) an example of that a triangle is placed, and (c) an example of that a triangle is picked. 


\section{OBJECT STATE ESTIMATOR TO SUPPORT THE GUIDANCE SYSTEM}

Given the specific physical configuration, an object state estimation method is proposed using color segmentation, object tracking, and a shape approximation algorithm.

\subsection{Object Extraction}

In order to minimize the tracking complexity and to make the tracking system robust, color segmentation in the Hue-Saturation-Value (HSV) color domain was applied first. Figure 2-(a) illustrates a training scenario as an example. Here, the considered object, in the following denoted as $\mathrm{T}_{1}$, is marked by a yellow box. As explained earlier, $\mathrm{T}_{1}$ 's initial position as well as its color are assumed to be known. The color segmentation result in the binary image is depicted in Figure 2-(b). Here, every pixel which represents the color of $\mathrm{T}_{1}$ is set to one, the residual to zero. After applying this segmentation, the remaining algorithm is based on the resulting binary image. Therefore, the original colors do not need to be considered anymore. Further, adding new colored triangles is very efficient since only the parameters of the color segmentation have to be adjusted.

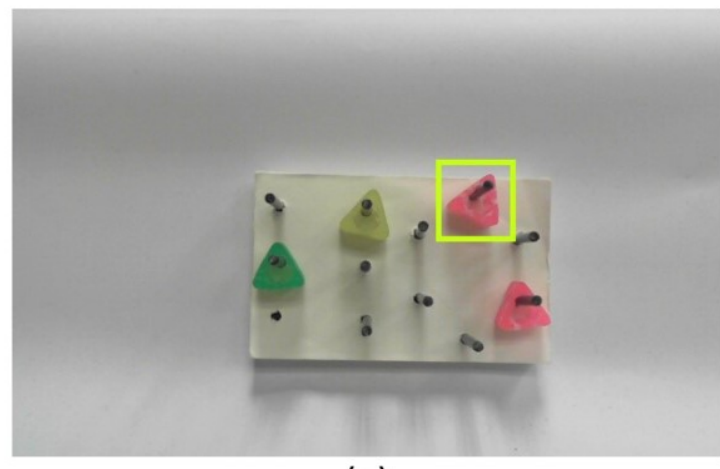

(a)

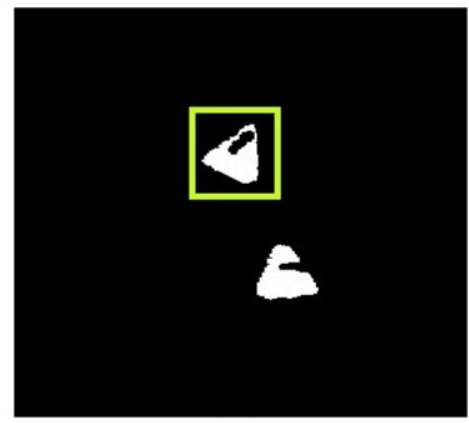

(c)

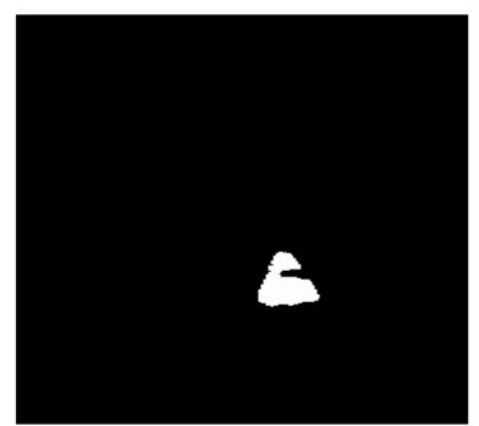

(d)

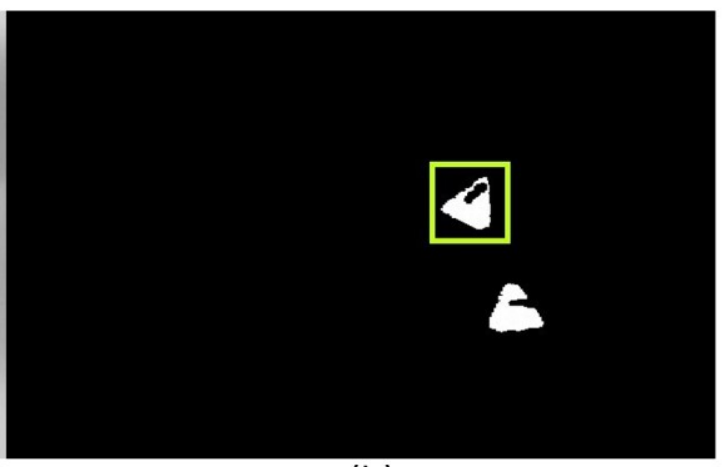

(b)

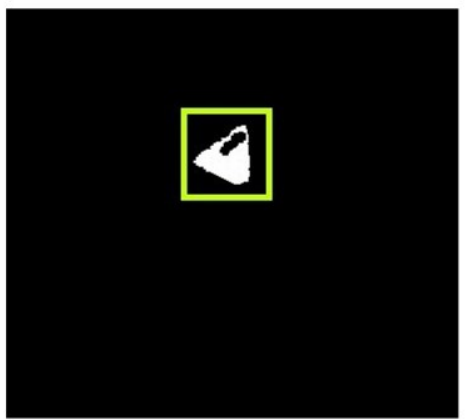

(e)

Figure 2: (a) an example configuration of peg transfer task, (b) a color segmented image, and (c)-(e) shows the background subtraction process: (c) a source binary image; (d) a background image; (e) a foreground image.

As introduced earlier, at least one specific color can be used twice which leads to a resulting binary image as shown in Figure 2-(b). Since there is an object with the same color of $\mathrm{T}_{1}$, which is denoted as $\mathrm{T}_{2}$ in the following, we cannot extract $\mathrm{T}_{1}$ by only applying color segmentation. To solve this issue, we introduce background subtraction in order to allow distinguishing the foreground, that only contains $\mathrm{T}_{1}$, from the background, containing $\mathrm{T}_{2}$. For each sub-task (e.g., transfer a designated triangle from a specific peg to another) given a PTT setup, a background image (i.e., a binary image which does not contain the designated triangle) is generated first as shown in Figure 2-(d). Given a source color segmented image (e.g., Figure 2(c)), a bounding box (2D size and location) information of the designated triangle is used to create background image. Based on the source binary image, every pixel in the area of $\mathrm{T}_{1}$ gets set to zero. In case 
the source image contains $\mathrm{T}_{1}$ and $\mathrm{T}_{2}$, the created background only shows the specific triangle, $\mathrm{T}_{2}$. Every pixel of the background is set to zero if there are no same color triangles. After applying background subtraction (i.e., bitwise operation to get a subtracted image (source color segmented image - background image)), the foreground image which only contains $\mathrm{T}_{1}$ is generated as shown in Figure 2-(e).

Using color segmentation and background subtraction requires some more considerations. First, as soon as the designated triangle is updated, caused by a changing training sub-task, we have to adjust the background. Second, the background subtraction can be slightly inaccurate. For example, a trainee can touch and turn $\mathrm{T}_{2}$ after the background was created. The result would be that $\mathrm{T}_{2}$ only gets subtracted partly and its corner would be visible. This phenomenon has to be considered and is compensated by the tracking approach discussed later in this paper.

\subsection{Object Tracking}

To track the designated triangle, we apply the Harris Corner Detection (Harris and Stephens 1988) to the created foreground, which results in a set of trackable features $Q$ as shown in Figure 3-(a). Here, each feature is represented by a blue point, drawn on the original frame. In the following, each feature is denoted as $q^{i}=\left(q_{x}^{i}, q_{y}^{i}\right) \in Q$ with $i \in \mathbb{N}$ and $0 \leq i<|Q|$ where $q_{x}^{i}$ and $q_{y}^{i}$ represent the $\mathrm{x}$ - and y- coordinate of $q^{i}$ in the 2D image plane and $|Q|$ the cardinality of $Q$. Let frame $f(k)$ be the current frame where the set $Q$ is found and $f(k+1)$ is the consecutive one. The new coordinates for every $q^{i} \in Q$ in $f(k+1)$ can be determined by using the Optical Flow (Beauchemin and Barron 1995). In this paper, we apply the Lucas Kanade Implementation (Lucas and Kanade 1981) since it offers robust results even for fast moving objects as well as efficient calculation.

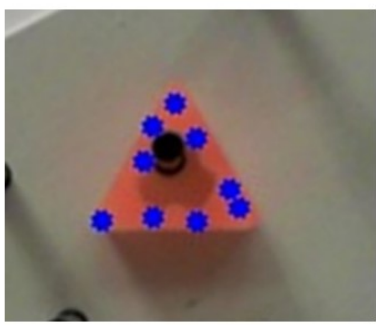

(a)

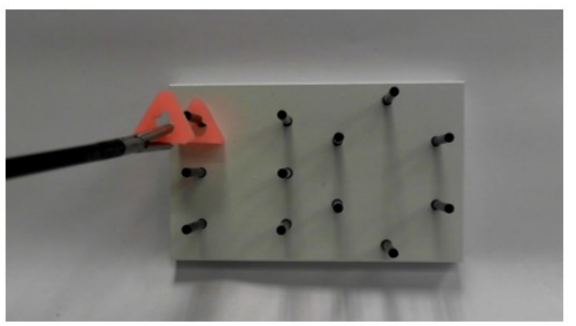

(b)

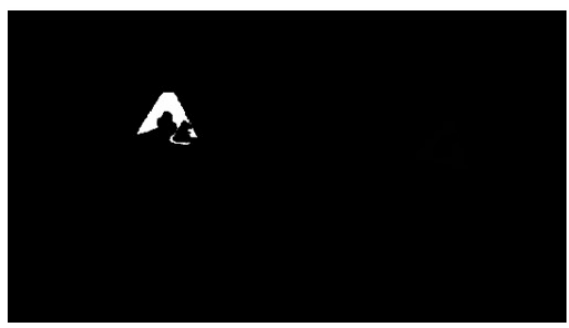

(c)

Figure 3: (a) trackable features by applying Harris Corner Detection, (b) an example of overlapping situation, and (c) a binary foreground image of the overlapping situation.

During the overlapping situation (Figure 3-(b) and Figure 3-(c)), however, the Optical Flow cannot be calculated $Q$ properly given the designated triangle $\left(\mathrm{T}_{1}\right)$ with a same color triangle $\left(\mathrm{T}_{2}\right)$. This results in deleting the affected points and, therefore, a decreasing cardinality of $Q$. To compensate for this phenomenon, the set of tracked features is updated during runtime by applying the Harris Corner Detection as needed. The previous section pointed out that the result of the background subtraction may be inaccurate if $\mathrm{T}_{2}$ gets turned after creating the background. Therefore, the new features should only be found in the current area of $\mathrm{T}_{1}$. To solve this issue, we define an area around $\mathrm{T}_{1}$ which is rectangular and can be described by its upper-left $\left(u_{x}, u_{y}\right)$ and lower-right corners $\left(l_{x}, l_{y}\right)$. Here, $u_{x}$ and $u_{y}$ denote the $\mathrm{x}-$ and $\mathrm{y}-$ coordinate of the upper-left corner and $l_{x}, l_{y}$ the coordinates of the lower-right corner. Further, let $Q_{x}$ and $Q_{y}$ be two sets which only contain the x-, respectively y-coordinates, of all $q^{i} \in Q$, respectively. The area around $\mathrm{T}_{1}$ can be defined as follows:

$$
\begin{aligned}
& \left(l_{x}, l_{y}\right)=\left(\max \left(Q_{x}\right)+z, \max \left(Q_{y}\right)+z\right), \\
& \left(u_{x}, u_{y}\right)=\left(\min \left(Q_{x}\right)-z, \min \left(Q_{y}\right)-z\right),
\end{aligned}
$$


where $\mathrm{z} \in \mathbb{R}^{+}$is a constant value. We use the value $\mathrm{z}$ to slightly increase the area around $\mathrm{T}_{1}$. Because features representing the edges or corners could be already deleted, this would result in the area being smaller than the actual designated triangle. Further, deleting the features on only one side of $\mathrm{T}_{1}$ would still lead to a rectangle where both edge lengths clearly differ. To avoid this, we assume that the area can be approximated by a square with the edge length $(L)$ defined as follows:

$$
L=\max \left(\operatorname{dist}\left(u_{x}, l_{x}\right), \operatorname{dist}\left(u_{y}, l_{y}\right)\right),
$$

where $\operatorname{dist}(\cdot, \cdot)$ is a function that calculates the Euclidean distance between two points. The square center $c^{0}$ is calculated as the mean of all tracked features and is given by

$$
c^{0}=\left(c_{x}^{0}, c_{y}^{0}\right)=\left(\frac{\sum q_{x}^{i}}{|Q|}, \frac{\sum q_{y}^{i}}{|Q|}\right),
$$

where $c_{x}^{0}$ and $c_{y}^{0}$ denote the $\mathrm{x}$ - and y-coordinate of the center. Even by limiting the area of the designated triangle $\left(\mathrm{T}_{1}\right)$, however, we cannot avoid detecting features of $\mathrm{T}_{2}$ if both same colored triangles are close or above each other. Thus, the features may not be updated properly under a certain condition. To enhance the update procedure, the position of $\mathrm{T}_{2}$ has to be determined as well. Applying a border following algorithm (Suzuki 1985) to the binary background image allows to detect the contours of $\mathrm{T}_{2}$ and, therefore, its position. Thus, the features only get updated if the distance between the center $c^{0}$ and $\mathrm{T}_{2}$ is larger than $L / 2$.

Even though the Lucas Kanade Implementation of the Optical Flow can handle fast movement, this approach can still fail. This could result in deleting all features before they can be updated. In case an object is lost during tracking, we apply a contour finding algorithm (Suzuki 1985) on the binary foreground image. Here, the largest found contour is assumed to represent $T_{1}$. To find new trackable features, the Harris Corner Detection is applied again. Calculating the center of this contour allows to set $c^{0}$, while the square length $L$ of this area is a predefined value.

\subsection{Triangle Approximation}

The triangle state can be determined by using three edges of the triangle. By adding the three edges between the corners, the scope can be calculated. The triangle state is redefined as follows:

$$
\text { Triangle state }=\left\{\begin{array}{rl}
\text { picked }(\mathrm{T}), & \text { scope } \geq \text { threshold } \\
\neg \text { picked }(\mathrm{T}), & \text { scope }<\text { threshold }
\end{array},\right.
$$

where the threshold describes the smallest scope from which beginning the object is assumed to be picked up. To calculate the scope, we present a triangle shape approximation method.

Given trackable features $Q$, three corners $\left(c^{1}, c^{2}, c^{3}\right)$ are estimated. The first corner $\left(c^{1}\right)$ of the triangle is determined as the $q^{i} \in Q$ which maximizes the distance $\operatorname{dist}\left(q^{i}, c^{0}\right)$ to the center $c^{0}$. Next, the feature with the largest distance from $c_{1}$ represents the second corner. Thus, the function $\operatorname{dist}\left(c^{1}, q^{i}\right)$ has to be maximized and the result is denoted as $c^{2}$. For determining the third corner $\left(c^{3}\right)$ we calculate the middle point $\left(m=\left(c^{1}+c^{2}\right) / 2\right)$ between $c^{1}$ and $c^{2}$ first. Finally, the third corner can be calculated as the feature which maximizes the function $\operatorname{dist}\left(m, q^{i}\right)$. Based on these three corners, the triangle and its scope can be defined. Figure 4 illustrates the overall triangle approximation procedure.

In the previous section, we defined upper-left and lower-right corners to enhance the tracking performance. These can be redefined using three corners $\left(c^{1}, c^{2}, c^{3}\right)$ as follows:

$$
\begin{aligned}
& \left(l_{x}, l_{y}\right)=\left(\max \left(c_{x}^{1}, c_{x}^{2}, c_{x}^{3}\right)+z, \max \left(c_{y}^{1}, c_{y}^{2}, c_{y}^{3}\right)+z\right), \\
& \left(u_{x}, u_{y}\right)=\left(\min \left(c_{x}^{1}, c_{x}^{2}, c_{x}^{3}\right)-z, \min \left(c_{y}^{1}, c_{y}^{2}, c_{y}^{3}\right)-z\right) .
\end{aligned}
$$




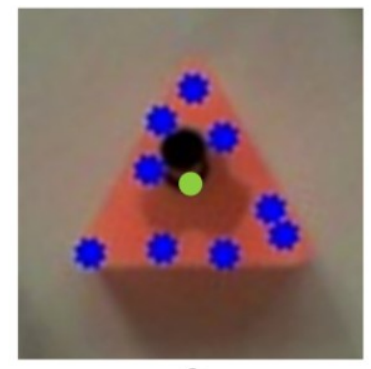

$c_{0}$

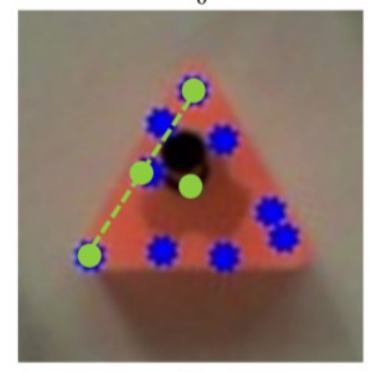

$m$

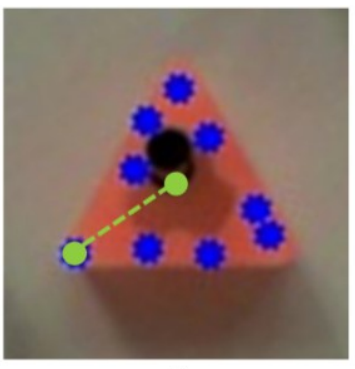

$c_{1}$

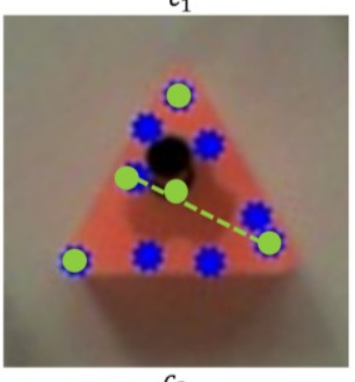

$c_{3}$

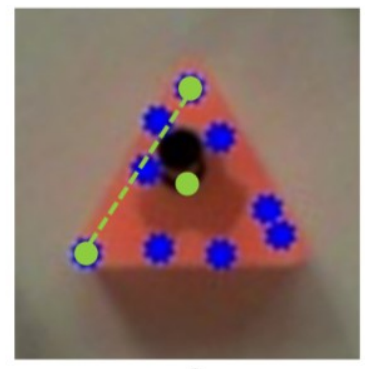

$c_{2}$

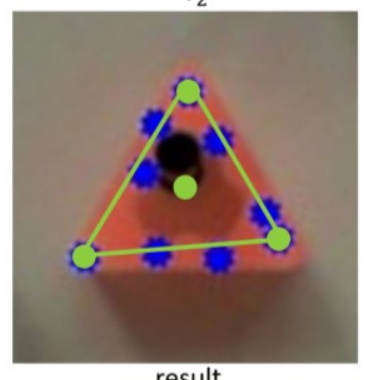

result

Figure 4: Triangle approximation procedure.

Also, we introduce a threshold $d_{\text {max }}$ which denotes the maximum allowable distance from a corner (i.e., one of $\left.\left(c^{1}, c^{2}, c^{3}\right)\right)$ to the center $\left(c^{0}\right)$ to solve a certain overlapping situation. In case the distance $\operatorname{dist}\left(q^{i}, c^{0}\right)$ is larger than $d_{\max }$, the considered feature $q^{i}$ cannot be assumed as a corner. Based on the experimental result, we set $d_{\max }=L / 2$.

When the designated triangle is not moving, updated features $Q$ allow a better triangle approximation if $q^{i} \in Q$ is closer to the current corners (at $f(k)$ ) than the previous ones (at $f(k-1)$ ). Thus, the distance between each corner and the center $c^{0}$ should be maximized, while the maximum allowed distance $d_{\max }$ must be considered, too. Let $c_{\text {old }}$ be a corner approximation of $f(k-1)$ and $c_{\text {new }}$ be the one based on the updated feature set from $f(k)$. If $\operatorname{dist}\left(c_{\text {old }}, c^{0}\right)<\operatorname{dist}\left(c_{\text {new }}, c^{0}\right)<d_{\max }$ is satisfied, the corner approximation $c_{\text {new }}$, which is based on the updated feature set, allows a better approximation than $c_{\text {old }}$. The reason is, its distance to the center $c^{0}$ is larger than the distance between $c^{0}$ and the previous corner $c_{\text {old }}$ and therefore more likely to be close to the corner we want to approximate. Thus, we use the updated feature set of frame $f(k)$ to approximate a corner in this case. Otherwise, the previous approximation (from frame $f(k-1))$ is used. If $\operatorname{dist}\left(c^{0}(k), c^{0}(k-1)\right)$ is greater than a threshold, then the triangle is considered to be moved. The threshold is determined based on an experimental result. The final updating rules for each corner are as follows:

$$
\begin{aligned}
& c^{1}(k)=\left\{\begin{array}{r}
c^{1}(k), \text { if } \operatorname{dist}\left(c^{1}(k-1), c^{0}(k)\right)<\operatorname{dist}\left(c^{1}(k), c^{0}(k)\right)<d_{\text {max }} \text { or moving } \\
c^{1}(k-1), \text { otherwise }
\end{array}\right. \\
& c^{2}(k)=\left\{\begin{array}{r}
c^{2}(k), \text { if } \operatorname{dist}\left(c^{2}(k-1), c^{0}(k)\right)<\operatorname{dist}\left(c^{2}(k), c^{0}(k)\right)<d_{\text {max }} \text { or } c^{1} \text { is updated }, \\
c^{2}(k-1), \text { otherwise }
\end{array}\right. \\
& c^{3}(k)=\left\{\begin{array}{c}
c^{3}(k), \text { if } \operatorname{dist}\left(c^{3}(k-1), c^{0}(k)\right)<\operatorname{dist}\left(c^{3}(k), c^{0}(k)\right)<d_{\text {max }} \text { or } c^{2} \text { is updated } \\
c^{3}(k-1), \text { otherwise }
\end{array}\right.
\end{aligned}
$$

\section{EXPERIMENTAL TEST RESULTS}

To evaluate the proposed state estimation, the algorithm is implemented in Python and runs on an Intel i7 $7500 \mathrm{U}$ CPU (2.7GHz and 2 cores with 4 threads). The sample videos (with 720P and $30 \mathrm{FPS}$ ) were taken 
by a Microsoft LifeCam studio. Besides the frame rate, the accuracy of predicting the triangle position as well as its state has to be tested. In our approach, we define the triangle position by using its center $\left(c^{0}\right)$. A bounding box could be calculated based on the triangle approximation, but it does not always contain accurate size information. For example, the bounding box would be very inaccurate during the overlapping situation. However, we could adjust the bounding box using a tuning parameter. This would improve the state estimation results but does not affect the accuracy of the triangle position estimation. Further, the proposed algorithm does not require the bounding box for the state prediction. Instead, the triangle shape is approximated in order to predict the object state. Thus, we measure the tracking accuracy by comparing the predicted center to the one we labeled manually. The shift is measured in pixels but can be converted into millimeters, since the actual triangle edge length and the camera matrix are known. Based on the known information, the edge length in pixels can be calculated and this relation can be used to convert every measured length in pixels. This offers more intuitive results. The state detection accuracy is measured by comparing the amount of correctly predicted frames to the wrong ones. Thus, it is important that the number of frames for each state is the same. Further, we especially consider the frames where the state should change. Therefore, the required amount of frames for changing each state is evaluated in detail.

The proposed method is tested in three different scenarios: Scenario 1) a simplified transfer task (i.e., transfer one triangle from one place to another given all different colored triangles) is evaluated with normal speed and no overlapping situation; Scenario 2) an overlapping situation is considered, as well as placing the considered triangle next to a same-colored object; Scenario 3) fast movement and re-detecting a lost object is evaluated. For all scenarios, the required computation time per one frame is about $9 \mathrm{~ms}$ on average. This leads to 110 FPS which fulfills the earlier introduced requirement of 100 FPS.

Scenario 1: For evaluating the simplified transfer task as shown in Figure 5-(a), we labeled the object position in every fifth frame given a considered scenario. Comparing all manually labeled centers to the predicted ones leads to an average shift of 2.7 pixels which equals less than $0.84 \mathrm{~mm}$. Here, we considered the frames where the triangle is placed as well as the ones where it is carried. Since we assumed to have a larger error while transferring the object, these frames where considered again separately. While the object is picked and transferred, the average error is 3 pixels and less than $0.92 \mathrm{~mm}$. Thus, moving the object does not lead to a significant change here. The accuracy of predicting the correct state in the considered scenario is $\sim 95 \%$ on average. The error turned out to depend on how well the triangle is placed on the boards. Figure 5-(b), for example, illustrates that the labeled triangle state is $\neg$ picked, since the triangle is on the peg and touched the board. However, the trainee still holds it while the object is slightly tilted and the right side of the triangular prism visible. This leads to a larger scope and assuming the triangle to be picked up. In this case, the number of wrongly predicted frames while placing the object was six. Due to using the 30 FPS camera, this leads to requiring 0.2 seconds delay until predicting the triangle to be placed, which is the average time duration, too.

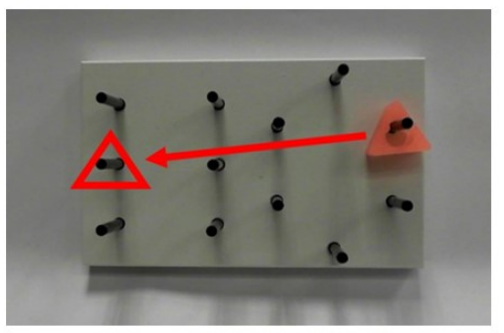

(a)

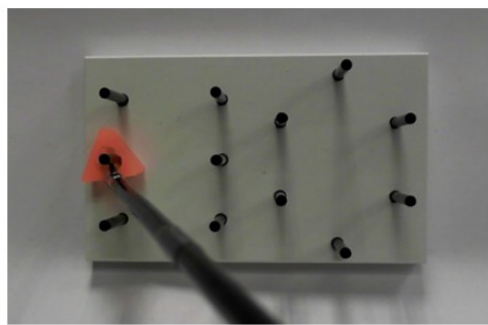

(b)

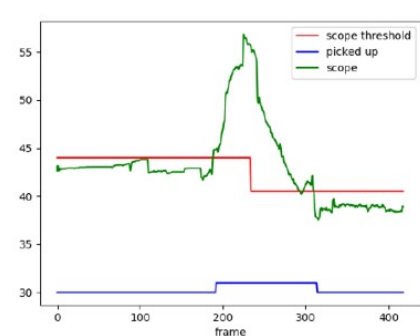

(c)

Figure 5: (a) a simplified task, (b) an example of the $\neg$ picked state, and (c) a result of state detection.

In Figure 5-(c), the current triangle scope and object state for each frame of the considered scenario can be seen. As the object gets picked in frame $\sim 190$ and placed on another peg in frame $\sim 310$, the scope turns out to differ depending on the object's location (i.e., the scope is smaller while the object is placed on the second peg). This phenomenon is caused by the camera being placed above the peg board center. In order 
to compensate the different scope sizes, we assigned an unique scope threshold to each peg. While the tracked object gets closer to the peg where it has to be placed, the threshold gets adjusted as can be observed in Figure 5-(c).

Scenario 2: During the overlapping situation as shown in Figure 6-(a), the tracking accuracy turned out to decrease. While both same colored triangles intersect, the average center shift is 11.6 pixels which equals about $3.5 \mathrm{~mm}$. This is more than three times the shift measured during the simplified transfer task. The reason is that carrying the designated triangle $\left(\mathrm{T}_{1}\right)$ above a same colored triangle $\left(\mathrm{T}_{2}\right)$ leads to losing features caused by the subtracted areas. This results in features which are not equally distributed on the designated triangle, as can be seen in Figure 6-(b). Here, only the right part of $\mathrm{T}_{1}$ intersected $\mathrm{T}_{2}$ and, therefore, only the affected features were deleted. As a result, the mean over the features is shifted to the upper-left area of $\mathrm{T}_{1}$. Note, Figure 6-(c) shows a frame after the overlapping situation where the features in the lower-right area of $\mathrm{T}_{1}$ are still deleted. As described earlier, we apply the Harris Corner Detection for finding new trackable features if required. Since a small set of features is unlikely to be well distributed over the area of $\mathrm{T}_{1}$, the set should be updated if the cardinality is smaller than a predefined threshold. At the same time, Figure 6-(c) shows that features should also be updated every couple frames. Here, deleting the tracked points in the lower right area doesn't lead to a significant change of the set's cardinality. As a result, the features don't get updated even though the current set doesn't allow an accurate center prediction. Updating the set every $n$-frames (i.e., the predefined value $n$ based on experimental results) leads to a steady adjustment of tracked points and avoids this phenomenon to occur in many consecutive frames.

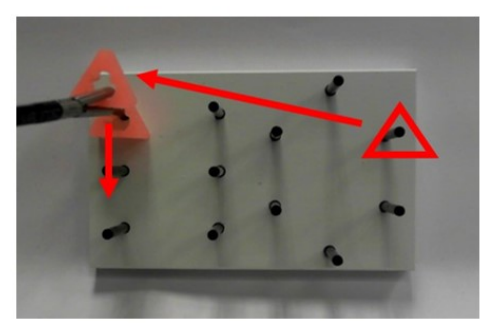

(a)

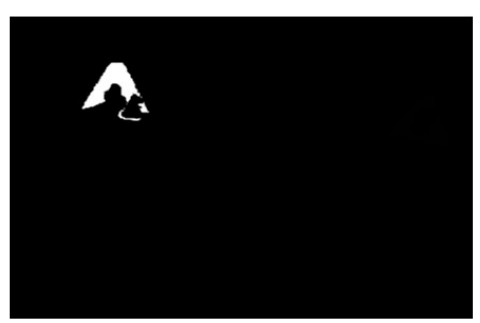

(b)

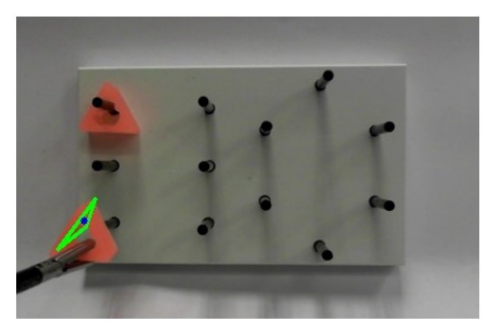

(c)

Figure 6: (a) an overlapping situation, (b) a foreground image, and (c) an example of the triangle approximation after the overlapping.

While placing $T_{1}$ next to $T_{2}$ as shown in Figure 7-(a), the state detection turned out to be slightly inaccurate due to deleted features if both triangles intersect. After a trainee picked $\mathrm{T}_{1}$, the tracked triangle is expected to move until it is placed again on a peg. While placing $T_{1}$ next to $T_{2}$, considering the movement $T_{1}$ as a state indicator turned out to be more robust, compared to the triangle shape size. If the movement of $\mathrm{T}_{1}$ between the frames is smaller than a threshold, it is expected to be $\neg$ picked. Otherwise, the current state is picked. The main challenge here is to find an appropriate motion threshold. On the one hand, small motion has to be detected, since this allows to accurately predict the state picked, even if the object is moved slowly. However, on the other hand, a small threshold turns out to be not very robust when it comes to placing the triangle. In case a trainee touches the object after placing it, this leads to object motion and $\mathrm{T}_{1}$ is still assumed to be picked. In our scenario, predicting the placement of the considered object requires 0.37 seconds delay. Here, the increased time duration, compared to previous scenarios, is caused by touching $\mathrm{T}_{1}$ after its placement while moving away the grasper.

Scenario 3: The tracking accuracy during fast movement is tested. In this scenario, the occurring speed of the tracked object is way faster than it would be expected during actual training. However, choosing an appropriate amount of pyramid levels for the Lucas Kanade Implementation leads to only losing a few features. While the average number of tracked features in each frame of the simplified transfer is $\sim 9.0$ features, the average cardinality of the feature set while testing the fast movement is $\sim 8.9$. Thus, the movement does not really have any impact considering the whole training scenario. Since features may get lost in single frames, the set gets more often updated in this scenario, compared to the simplified transfer. 
As a result, considering only the frames where the object moves fast, the average amount of features even increases to $\sim 9.5$ features. At the same time, the set of tracked points is updated regularly. Thus, the fast movement results in an average center prediction error of 5.54 pixels which equals less than $1.44 \mathrm{~mm}$. Compared to the simplified transfer, this does not lead to a significant accuracy loss. However, detecting lost features should also be tested. For this purpose, the number of levels was decreased which leads to losing more tracked points at a time and causes losing the tracked object. In this scenario, the average number of tracked features decreases to $\sim 5.9$, respectively $\sim 5.5$ when only considering the frames while the object moves fast. The evaluation showed, that the triangle is re-detected again in the same frame as it was lost as shown in Figure 7-(b). At the same time, applying the contour finding algorithm does not lead to a significant change of the frame rate.

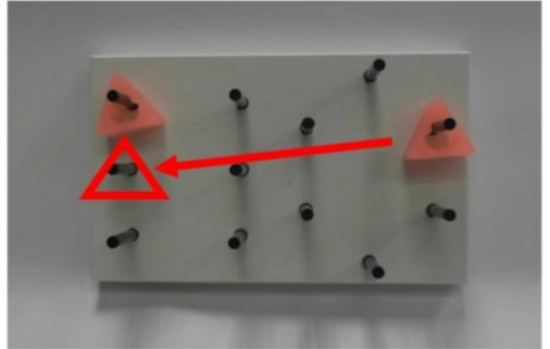

(a)

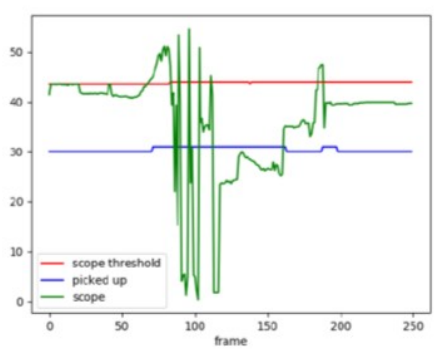

(b)

Figure 7: (a) an example of placing the designated triangle next to the same colored triangle and (b) a result of state detection when a triangle moves rapidly (an object is lost in frame 90, 98 and 105).

\section{CONCLUSION}

In this paper, we presented an object tracking and state estimation method using color segmentation, background subtraction, and shape approximation to support the guidance system of CAST. The proposed method which is a robust and cost effective solution has an ability to support real-time application given a PTT setup. Running on a CPU, the reached frame rate is about 109 FPS on average, which is more computationally efficient than the computation speed of SSSD. Also, the average center shift is less than $1.5 \mathrm{~mm}$ and the delay of detecting the correct state is about 0.2 seconds. Therefore, this approach highly improves the efficiency without using an expensive GPU. At the same time, the predicted state as well as $2 \mathrm{D}$ position can be estimated accurately. Thus, the experimental test results show that this approach can be assumed to offer enough precision to evaluate a trainee's performance and provide appropriate guidance.

By combining this proposed triangle state estimation method with a grasper state estimation (e.g., open/close detection using a bend sensor) and its position information, all the actions of PTT will be recognized and the guidance system will provide a proper information (e.g., suggest recommended trajectory using augmented reality (AR) overlays and applying force feedback to assist a trainee). Also, an objective evaluation system will use the proposed method to evaluate the performance of each action by detecting transition conditions (e.g., measure completion time for each action).

Knowledge of the state of instruments and objects is beneficial not only for simulation-based surgical training but also could ultimately be used in real-time in the operating room. As technology becomes more and more ubiquitous as an assistive element in medicine, robust methods must be in place that ensure the safety and reliability of the assistance that it will offer.

\section{ACKNOWLEDGMENTS}

This material is based upon work supported by the National Science Foundation under Grant Number 1622589 "Computer Guided Laparoscopy Training". Any opinions, findings, and conclusions or 
recommendations expressed in this material are those of the authors and do not necessarily reflect the views of the National Science Foundation.

\section{REFERENCES}

Beauchemin, S. S., and J. L. Barron. 1995. "The Computation of Optical Flow.” ACM Computing Surveys (CSUR) 27 (3): 433-66.

Duffy, A. J., N. J. Hogle, H. McCarthy, J. I. Lew, A. Egan, P. Christos, and D. L. Fowler. 2005. "Construct Validity for the LAPSIM Laparoscopic Surgical Simulator." Surgical Endoscopy 19 (3): 401-5. doi:10.1007/s00464-004-8202-9.

Escamirosa, Fernando Pérez, Ricardo Manuel Ordorica Flores, Ignacio Oropesa García, Cristian Rubén Zalles Vidal, and Arturo Minor Martínez. 2015. "Face, Content, and Construct Validity of the EndoViS Training System for Objective Assessment of Psychomotor Skills of Laparoscopic Surgeons.” Surgical Endoscopy 29 (11): 3392-3403. doi:10.1007/s00464-014-4032-6.

"Fundamentals of Laparoscopic Surgery." 2019. Accessed December 8. http//www.flsprogram.org/.

Harris, C., and M. Stephens. 1988. "A Combined Corner and Edge Detector." In Alvey Vision Conference, $147-51$.

Hernansanz, Albert, Davide Zerbato, Lorenza Gasperotti, Michele Scandola, Paolo Fiorini, and Alicia Casals. 2012. "Improving the Development of Surgical Skills with Virtual Fixtures in Simulation." In International Conference on Information Processing in Computer-Assisted Interventions, 157-66. doi:10.1007/978-3-642-30618-1_16.

Hong, Minsik, and Jerzy W. Rozenblit. 2016a. "A Haptic Guidance System for Computer-Assisted Surgical Training Using Virtual Fixtures." In IEEE International Conference on Systems, Man, and Cybernetics, 2230-35. doi:10.1109/SMC.2016.7844570.

Hong, Minsik, and Jerzy W. Rozenblit. 2016b. "Modeling of a Transfer Task in Computer Assisted Surgical Training." In Proceedings of the Symposium on Modeling and Simulation in Medicine. Society for Computer Simulation International, 794-99.

Hong, Minsik, Jerzy W. Rozenblit, and Allan J. Hamilton. 2017. “A Simulation-Based Assessment System for Computer Assisted Surgical Trainer." In Proceedings of the Symposium on Modeling and Simulation in Medicine. Society for Computer Simulation International, 834-44.

Howard, Thomas, and Jerome Szewczyk. 2014. "Visuo-Haptic Feedback for 1-D Guidance in Laparoscopic Surgery." In 5th IEEE RAS/EMBS International Conference on Biomedical Robotics and Biomechatronics, 58-65. doi:10.1109/BIOROB.2014.6913752.

Lahanas, Vasileios, Constantinos Loukas, Nikolaos Smailis, and Evangelos Georgiou. 2015. "A Novel Augmented Reality Simulator for Skills Assessment in Minimal Invasive Surgery." Surgical Endoscopy 29 (8): 2224-34. doi:10.1007/s00464-014-3930-y.

Lau, W. Y., C. K. Leow, and Arthur K.C. Li. 1997. "History of Endoscopic and Laparoscopic Surgery." World Journal of Surgery 21 (4): 444-53. doi:10.1007/PL00012268.

Lucas, Bruce D, and Takeo Kanade. 1981. "An Iterative Image Registration Technique with an Application to Stereo Vision." In Proceedings DAPRA Image Understanding Workshop, 121-30.

Napalkova, Liana, Jerzy W. Rozenblit, George Hwang, Allan J. Hamilton, and Liana Suantak. 2014. "An Optimal Motion Planning Method for Computer-Assisted Surgical Training." Applied Soft Computing Journal 24 (April 2015): 889-99. doi:10.1016/j.asoc.2014.08.054.

Oropesa, Ignacio, Patricia Sánchez-González, Pablo Lamata, Magdalena K. Chmarra, José B. Pagador, Juan A. Sánchez-Margallo, Francisco M. Sánchez-Margallo, and Enrique J. Gómez. 2011. "Methods and Tools for Objective Assessment of Psychomotor Skills in Laparoscopic Surgery." Journal of Surgical Research 171 (1): e81-95. doi:10.1016/j.jss.2011.06.034. 
Peng, Kuo Shiuan, Minsik Hong, Jerzy Rozenblit, and Allan J. Hamilton. 2019. "Single Shot State Detection in Simulation-Based Laparoscopy Training." 2019 Spring Simulation Conference, SpringSim 2019. SCS. doi:10.23919/SpringSim.2019.8732863.

Rao, Aakarsh, Michael Valenzula, and Jerzy W. Rozenblit. 2017. "Safety-Assured Offline-Online Path Planning with Reduced Storage Requirements in Simulated Surgical Training." In , 879-90.

Rosen, Jacob, Jeffrey D. Brown, Marco Barreca, Lily Chang, Blake Hannaford, and Mika Sinanan. 2002. "The Blue DRAGON - A System for Monitoring the Kinematics and the Dynamics of Endoscopic Tools in Minimally Invasive Surgery for Objective Laparoscopic Skill Assessment." In Studies in Health Technology and Informatics, 85:412-18. doi:10.3233/978-1-60750-929-5-412.

Rozenblit, J W, C Feng, M Riojas, L Napalkova, A J Hamilton, M Hong, P Berthet-Rayne, et al. 2014. "The Computer Assisted Surgical Trainer: Design, Models, and Implementation." In Proceedings of the 2014 Summer Simulation Multiconference, 211-20. doi:10.1145/2254556.2254608.

Salkini, Mohamad W., Charles R. Doarn, Nicholai Kiehl, Timothy J. Broderick, James F. Donovan, and Krishnanath Gaitonde. 2010. "The Role of Haptic Feedback in Laparoscopic Training Using the LapMentor II.” Journal of Endourology. doi:10.1089/end.2009.0307.

Soper, N J, and G M Fried. 2008. "The Fundamentals of Laparoscopic Surgery: Its Time Has Come." Bull Am Coll Surg 93 (9): 30-32.

Stylopoulos, N., S. Cotin, S. K. Maithel, M. Ottensmeyer, P. G. Jackson, R. S. Bardsley, P. F. Neumann, D. W. Rattner, and S. L. Dawson. 2004. "Computer-Enhanced Laparoscopic Training System (CELTS): Bridging the Gap.” Surgical Endoscopy 18 (5): 782-89. doi:10.1007/s00464-003-8932-0.

Suzuki, Satoshi. 1985. "Topological Structural Analysis of Digitized Binary Images by Border Following." Computer Vision, Graphics, and Image Processing 30 (1): 32-46.

Tagawa, Kazuyoshi, Hiromi T. Tanaka, Yoshimasa Kurumi, Masaru Komori, and Shigehiro Morikawa. 2013. "Laparoscopic Surgery Simulator Using First Person View and Guidance Force." Studies in Health Technology and Informatics 184 (February): 431-35. doi:10.3233/978-1-61499-209-7-431.

Wagner, Adam, and Jerzy W. Rozenblit. 2017. "Augmented Reality Visual Guidance for Spatial Perception in the Computer Assisted Surgical Trainer." In Proceedings of the Symposium on Modeling and Simulation in Medicine. Society for Computer Simulation International, 855-66.

\section{AUTHOR BIOGRAPHIES}

KAI MEISNER is a master's student in Computer Science at the University of the Armed Forces in Munich, Germany. He visited the University of Arizona as part of an exchange program, where he worked at the Model-Based Design Laboratory. His email address is kai.meisner@googlemail.com.

MINSIK HONG is a Research Assistant Professor at the University of Arizona. He holds a Ph.D. in Electrical and Computer Engineering from the University of Arizona. His research interests are robotics, control system, fuzzy theory, and modeling and simulation for medical devices. His email address is mshong@email.arizona.edu.

JERZY W. ROZNEBLIT is University Distinguished Professor, Raymond J. Oglethorpe Endowed Chair in the Electrical and Computer Engineering (ECE) Department, with a joint appointment as Professor of Surgery in the College of Medicine at the University of Arizona. During his tenure at the University of Arizona, he established the Model-Based Design Laboratory with major projects in design and analysis of complex, computer-based systems, hardware/software codesign, and simulation modeling. He presently serves as Director of the Life-Critical Computing Systems Initiative, a research enterprise intended to improve the reliability and safety of technology in healthcare and life-critical applications. His email address is jr@ece.arizona.edu. 